\title{
Phone Interview Hang Ups During Introduction: A Growing Challenge
}

Barbara C O'Hare*, Diana Buck ${ }^{\dagger}$

Tags: survey practice

\section{Survey Practice}

Vol. 2, Issue 3, 2009

Phone Interview Hang Ups During Introduction: A Growing Challenge

For anyone running a call center or overseeing phone surveys, respondent terminations by hanging up on the interviewer in the first few seconds of the interview are a serious concern. This affects survey completion rates, call throughput rates, and measurement reliability. There is little, if any, time to overcome objections or to assess anything about who has been contacted. Since the early work of Oksenberg and Cannell (1986; 1988), there have been relatively few published studies on what seems to be, among conversations with practitioners, a common problem.

The data presented here describes the incidence and characteristics of hang-ups-during-introduction (sometimes called HUDIs), as experienced by Arbitron Inc. The data are based on the results of standard interviewer monitoring of $5 \%$ of all calls and reflect hang-up rates during the first five sentences of the survey, before the first data collection question, whether the respondent has said anything at all or not.

As seen in Figure 1, the incidence of initial hang-ups has been growing steadily over the past five years. 


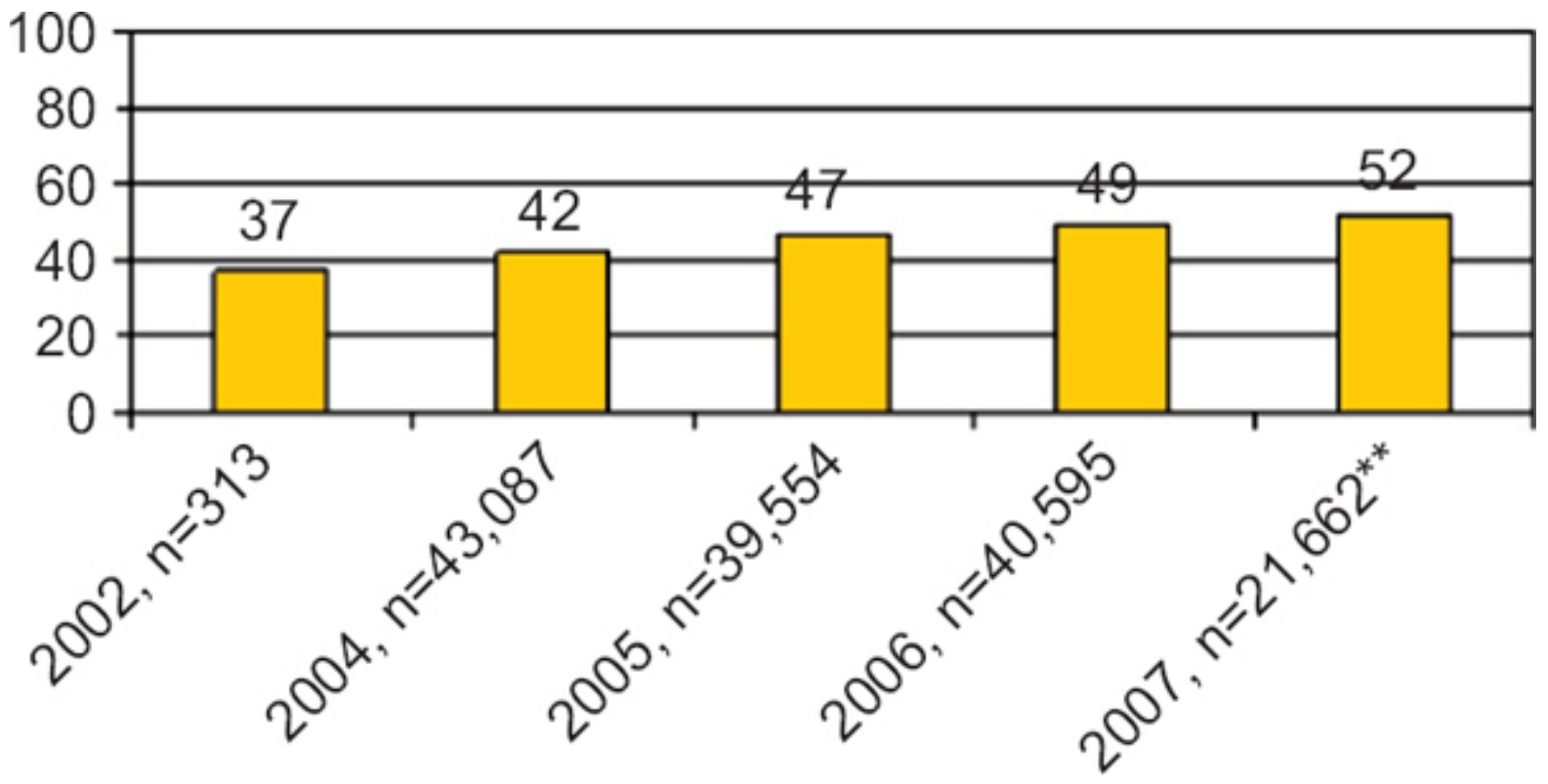

Figure 1 Incidence of Hang-Ups During Introduction as Percent of Contacts.

Further, $84 \%$ of the hang-ups occur during the first three sentences of the introduction. (Figure 2)

\section{घOverall $\square$ First contact $\square$ Conversion}

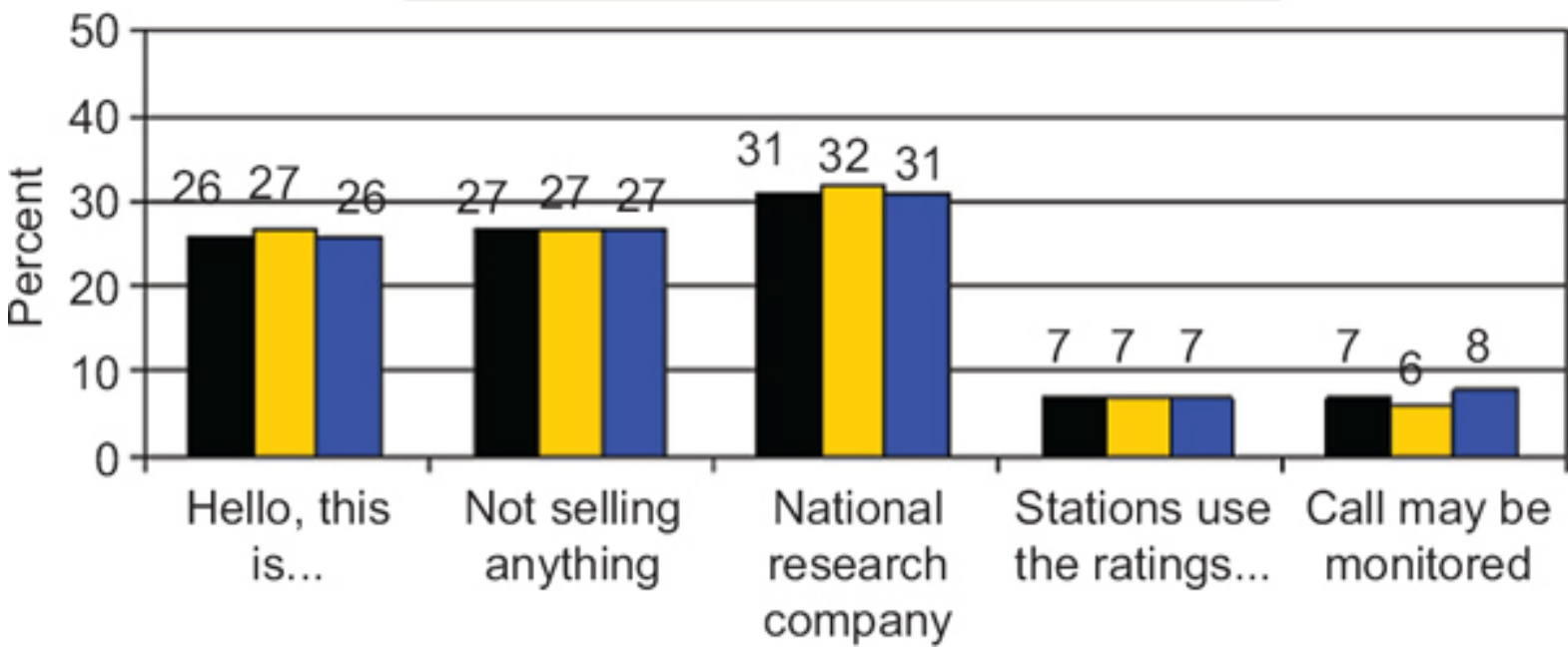

Figure 2 Percent of Hang-Ups by Sentence of Hang-Up.

Examination of known characteristics of the phone number found that the small town Midwest stereotype holds. The incidence of HUDIs was significantly lower in the Midwest $(46 \%, \mathrm{p}<0.01)$ than in the South $(49 \%)$, Northeast (52\%) and West (53\%). In the smallest towns of less than 50,000 population, the hang-up rate (47\%) was significantly lower than all other larger metropolitan areas. Phone numbers with addresses, which were sent pre-alert mailings before the first call, show a lower incidence of hang-ups ( $48 \%$ vs. $52 \%$, 
$\mathrm{p}<0.01)$. This is likely the result of both the mailing and other characteristics of the household that differ by address availability.

In an attempt to determine if there were characteristics of smaller units of geography that were predictors of hang-up rates, a logistic model was fit that included 2000 census block group data for all phone numbers for which we had addresses. None of the small area geography indicators, which included home value, income and race added to the significant predictors of time of day of the call, whether the call was a first contact or conversion call, census region and metro rank.

Last, looking at the final outcome of the HUDI cases, we found that $90 \%$ of the hang-ups resulted in a final disposition as a refusal.

\section{CONCLUSIONS}

Although we don't know much about the household when they hang up on us, we know that this group of respondents is particularly challenging and may warrant special procedures to overcome their reluctance to participate. While considerable literature exists experimenting with introductions, including what to say, whether they should be standardized, the value of caller ID and the effects of interviewer characteristics, there is still much to be learned. From the survey researcher and the practitioner perspectives, there are real costs in hang-ups during introductions.

The authors would welcome comments on the experience of other survey organizations in the incidence of HUDIs and what they have tried to attack this particular challenge. 